\title{
Absence of significant associations between four AKT1 SNP markers and schizophrenia in the Taiwanese population
} Yu-Li Liua, Cathy Shen-Jang Fann ${ }^{b}$, Chih-Min Liua ${ }^{a}$, Jer-Yuarn Wuc, Shuen-lu Hung ${ }^{c}$, Hung-Yu Chan ${ }^{d}$, Jiahn-Jyh Chen ${ }^{d}$, Chia-Ching Pan ${ }^{b}$, Shih-Kai Liư ${ }^{a}$, Ming H. Hsieha, Tzung-Jeng Hwang ${ }^{a}$, Wen-Chen OuYang, ${ }^{\mathrm{d}, \mathrm{e}}$, Chun-Ying Chen ${ }^{\dagger}$, Jin-Jia Lin ${ }^{9}$, Frank Huang-Chih Chou ${ }^{h}$, Ching-Mo Chueh', Wei-Ming Liu', Ming-Ming Tsuang ${ }^{k}$, Stephen V. Faraone', Ming T. Tsuang ${ }^{\mathrm{m}, \mathrm{n}, \mathrm{o}, \mathrm{p}}$, Wei J. Chen ${ }^{q}$ and Hai-Gwo Hwa ${ }^{a, q, r}$

AKT1 (V-akt murine thymoma viral oncogene homolog 1) is a protein kinase isoform of AKT. Five single-nucleotide polymorphisms, rs3803300, rs1130214, rs3730358, rs2498799 and rs2494732, at the genomic region of AKT1 have been reported to be significantly associated with schizophrenia. We tested for the presence of these five single-nucleotide polymorphisms in a Taiwanese population by genotyping 218 co-affected schizophrenia families. Both single locus and haplotypes analyses showed no association of these single-nucleotide polymorphisms with schizophrenia. These findings fail to support AKT1 as a susceptibility gene for schizophrenia in the Taiwanese population. Psychiatr Genet 16:39-41 (c) 2006 Lippincott Williams \& Wilkins.

Psychiatric Genetics 2006, 16:39-41

Keywords: protein kinase, schizophrenia, Taiwanese families, V-akt murine thymoma viral oncogene homolog 1

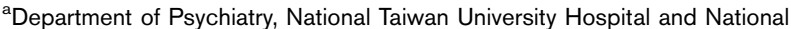

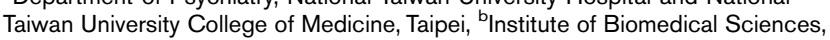

\section{Introduction}

AKT1 (V-akt murine thymoma viral oncogene homolog 1) is an isoform of serine/threonine protein kinase AKT (also known as protein kinase B), which was isolated from an AKR mouse thymoma cell line transforming with murine retrovirus AKT8 (Staal, 1987).

The AKT1 gene was first reported as a susceptibility gene in schizophrenia by Emamian et al. (2004), who found a significant reduction of AKT1 in the viral-transformed peripheral lymphocytes of schizophrenic patients, and also confirmed this result in post-mortem studies of the human brain frontal cortex and hippocampus. Besides the proteomic findings, five single-nucleotide polymorphisms (SNPs) of AKT1 markers located at the chromosome $14 q 32.32$ spanning from the promoter to the intron 11 were genotyped in 265 schizophrenia proband families. Their study revealed significant haplotype associations close to the promoter region of the AKT1 gene. The
Academia Sinica, Taipei, 'National Genotyping Center, Institute of Biomedical Sciences, Academia Sinica, Taipei, ${ }^{\mathrm{d}}$ Taoyuan Psychiatric Center, Taoyuan, ${ }^{\mathrm{e}} \mathrm{Chia}-$ Nan Psychiatric Center, Tainan, 'Tsaotun Psychiatirc Center, Tsaotun,

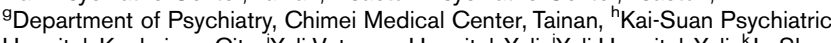
Hospital, Kaohsiung City, 'Yuli Veterans Hospital, Yuli, ${ }^{\text {YYuli Hospital, Yuli, }{ }^{\mathrm{K}} \mathrm{Ju} \text {-Shan }}$ Mental Hospital, Taoyuan, Taiwan, 'Medical Genetics Research Center and Department of Psychiatry and Behavioral Sciences, SUNY Upstate Medical University, Syracuse, New York, mHarvard Institute of Psychiatric Epidemiology and Genetics, "Departments of Epidemiology, ${ }^{\circ}$ Psychiatry, Harvard University, Boston, Massachusetts, PInstitute of Behavioral Genomics, University of California, San Diego, California, USA, IInstitute of Epidemiology, College of Public Health and 'Department of Psychology, College of Science, National Taiwan University, Taipei, Taiwan

Correspondence and requests for reprints to Hai-Gwo Hwu, MD, Department of Psychiatry, National Taiwan University Hospital, No. 7, Chung San South Road, Taipei 100 Taiwan

Tel: 886223123456 ext 6657; fax: 88622331 4775; e-mail:

haigohwu@ha.mc.ntu.edu.tw

Sponsorship: This study was supported by grants from the National Science Council, Taiwan (NSC-91-3112-B-002-011; NSC-92-3112-B-002-019; NSC93-3112-B-002-012), the National Health Research Institute, Taiwan (NHRI-908825PP; NHRI-EX91, 92, 93-9113PP) and the National Institute of Health, Bethesda, Maryland, USA ( IRO1 MH59624-01).

Received 21 July 2005 Accepted 28 September 2005

substrate of AKT1, glycogen synthase kinase- $3 \beta$, decreased its phosphorylation of the serine 9 residue. The mRNA levels of AKT1 at the dorsolateral prefrontal cortex were also decreased in the schizophrenia patients (Kozlovsky et al., 2004). Two separate genotyping studies of these five SNPs of AKT1 in the Japanese population, however, revealed contradictory results (Ikeda et al., 2004; Ohtsuki et al., 2004). In order to determine whether the AKT1 gene is a schizophrenia-susceptibility gene in the Taiwanese population, we genotyped these five SNPs in 218 affected sib-pair schizophrenia families.

\section{Study participants and methods}

This research project was approved by the Institutional Review Board of the National Taiwan University Hospital. All genomic DNA samples were collected from families with at least two affected siblings after obtaining written, informed consent. The participants were recruited from two research programs: the Multidimensional 
Psychopathology Study of Schizophrenia (MPSS) (Hwu et al., 2002) from 1993 to 2001 and the Taiwan Schizophrenia Linkage Study (TSLS) (Hwu et al., 2005) from 1998 to 2002. The 86 families of MPSS participants were interviewed by the research psychiatrists using the Psychiatrist Diagnostic Assessment (PDA) (Hwu, 1999). The 132 TSLS families were interviewed by well trained assistants using the Mandarin Chinese version of the Diagnostic Interview for Genetic Studies (DIGS) (Chen, 1999). For both studies, the final diagnostic assessment was formulated by integrating either the PDA or the DIGS data with clinical information from medical records using the Specialist Diagnostic Assessment Sheet, based on the criteria of the Diagnostic and Statistical Manual of Mental Disorders, 4th edition.

A total of 218 families participated in this study. Among these families, there were 199 complete families (at least one parent and one affected sibling), and 19 families without parents participated. A total of 1006 individuals were investigated, but only 854 participants had a DNA sample and were genotyped. All affected individuals were diagnosed as having schizophrenia only. No schizoaffective or non-affective psychotic disordered patients were included.

All SNP markers were genotyped by the method of matrix-assisted laser desorption/ionization-time of flight mass spectrometry (MALDI-TOF MS). A DNA fragment (100-300 bp) encompassing the SNP site was amplified using the polymerase chain reaction GeneAmp 9700 thermocycler (Applied Biosystems, Foster City, California, USA) according to the manufacturer's instructions. After polymerase chain reaction amplification and neutralization of the deoxynucleotide triphosphate (dNTP), the primer extension was performed by adding the probe, Thermo Sequenase (Amersham Pharmacia, Piscataway, New Jersey, USA) and appropriate dideoxynucleotide triphosphate (ddNTP)/dNTP mixture. Different extension products were differentiated by mass through MALDI-TOF.
We used the procedure ALLELE in SAS/GENETICS release 8.2 (SAS Institute, 2002) to assess Hardy-Weinberg equilibrium. Family relationships were verified by PEDCHECK version 1.1 (O'Connell and Weeks, 1998) and UNKNOWN version 5.23 (Terwilliger and Ott, 1994) to detect deviations from Mendelian inheritance. Both singlepoint and haplotype association analyses were carried out using TRANSMIT version 2.5.4 (Clayton, 1999).

\section{Results}

All of the family relationships and genotypes were verified by checking Mendelian inheritance and using the procedure ALLELE in SAS/GENETICS release 8.2 for Hardy-Weinberg equilibrium. The SNP markers were all compatible with the Hardy-Weinberg equilibrium distribution, except for SNP1 (rs3803300). The other four SNPs (SNP 2-SNP 5) were validated and showed a minor allele frequency greater than $10 \%$ and a missing rate of less than $2 \%$ in this Taiwanese cohort (Table 1 ).

We used haploview software to construct haplotype blocks constituted by 'strong LD' markers according to the criteria proposed by Gabriel et al. (2002). The results show that only one haplotype block formed by SNP4 and SNP5 $\left(D^{\prime}=0.96\right)$ was found among the four markers. The TRANSMIT program version 2.5.4 (Clayton, 1999), which can utilize data from all families even when parental genotypes are unknown, was used to analyze the associations between single SNPs, the haplotypes and schizophrenia. No significant association was found between a single locus or haplotype and schizophrenia (Table 1). Similar results were also obtained from single locus: SNP $2(n=35, Z=-0.277, P=0.782)$, SNP 3 $(n=21, Z=-0.14, P=0.889), \mathrm{SNP} 4(n=81, Z=0.32$, $P=0.748)$, and SNP $5(n=74, Z=0.37, P=0.714)$, and from haplotypes: $\mathrm{C}-\mathrm{T}(n=85, Z=0.8, P=0.422)$, T-C $(n=79, Z=-0.45, P=0.65), \mathrm{C}-\mathrm{C}(n=50, Z=-0.449$, $P=0.65)$, and T-T $(n=1)$ association analyses performed by family-based tests, using the FBAT program (Horvath et al., 2004).

Table 1 Frequencies of the single nucleotide polymorphisms and haplotypes of the AKT1 gene and associations (analyzed by Transmit program 2.5.4.) with schizophrenia

\begin{tabular}{|c|c|c|c|c|c|c|c|c|c|c|c|}
\hline \multirow[b]{2}{*}{$\begin{array}{l}\text { Report SNP SNP_ID } \\
\text { (primer ID) }\end{array}$} & \multirow[b]{2}{*}{ Position } & \multirow[b]{2}{*}{ Genetic region } & \multirow[b]{2}{*}{ Allele type } & \multirow[b]{2}{*}{ MF } & \multirow{2}{*}{$\begin{array}{c}\text { HW test } \\
P\end{array}$} & \multicolumn{2}{|c|}{$\begin{array}{l}\text { Schizophrenia } \\
(n=214)\end{array}$} & \multicolumn{2}{|c|}{ Haplotypes } & \multicolumn{2}{|c|}{$\begin{array}{c}\text { Schizophrenia } \\
\quad(n=214)\end{array}$} \\
\hline & & & & & & $\chi^{2}$ & $P$ & $\begin{array}{c}\text { Haplotype } \\
\text { (rs2498799- } \\
\text { rs2494732) }\end{array}$ & $\begin{array}{l}\text { Haplotype } \\
\text { frequency }\end{array}$ & $\chi^{2}$ & $P$ \\
\hline $\begin{array}{l}\text { SNP2 rs1130214 } \\
(9128)\end{array}$ & 10323141 & AKT1 (promoter) & $\mathrm{C} / \mathrm{A}$ & 0.1098 & 0.6157 & 0.177 & 0.674 & $\mathrm{C}-\mathrm{T}$ & 0.5594 & 1.635 & 0.201 \\
\hline $\begin{array}{l}\text { SNP3 rs3730358 } \\
(9127)\end{array}$ & 10321808 & AKT1 (intron 3) & $\mathrm{G} / \mathrm{A}$ & 0.0538 & 0.2056 & 0.267 & 0.605 & $\mathrm{~T}-\mathrm{C}$ & 0.2755 & 0.124 & 0.724 \\
\hline $\begin{array}{l}\text { SNP4 rs2498799 } \\
(9132)\end{array}$ & 10321157 & AKT1 (exon 9) & $\mathrm{T} / \mathrm{C}$ & 0.4414 & 0.6591 & 0.829 & 0.363 & $\mathrm{C}-\mathrm{C}$ & 0.1573 & 1.283 & 0.257 \\
\hline $\begin{array}{l}\text { SNP5 rs2494732 } \\
(9131)\end{array}$ & 10321086 & AKT1 (intron 11) & $\mathrm{C} / \mathrm{T}$ & 0.2966 & 0.9365 & 0.464 & 0.496 & T-T & 0.0077 & 2.834 & 0.092 \\
\hline
\end{tabular}

MF, minor allele frequency; HW, Hardy-Weinberg; $n$, number of families. 


\section{Discussion}

Although chromosome $14 \mathrm{q}$ has been reported in a genomewide scan as a susceptible locus for schizophrenia (Bailer et al., 2000), AKT1 was the first candidate gene for schizophrenia in the chromosome 14q32.32 region reported from SNP2-SNP3-SNP4 (T-C-G) haplotype association analyses (Emamian et al., 2004). The single locus result was similar to the result obtained from this Taiwanese sample. The minor allele frequencies in the study by Emamian $e$ al. and us are the following: SNP2 0.29 and 0.11 , SNP3 0.17 and 0.054, SNP4 0.25 and 0.44, and SNP5 0.44 and 0.3, respectively. While Emamian $e$ al. (2004) reported that the risk haplotypes in the SNP2SNP3 had a linkage disequilibrium of around 0.67 , this study found an SNP4-SNP5 block with $D^{\prime}$ of 0.98 . This result suggests inheritance differences between different ethnic groups.

If the AKT1 gene has a major role in schizophrenia susceptibility, we would expect studies to consistently demonstrate its association with the disease development among members of an ethnic group. In the Japanese population, however, inconsistent results for this association have been reported (Ikeda et al., 2004; Ohtsuki et al., 2004). The present study also could not confirm the significance of Emamian et al.'s (2004) finding of an association between AK'T1 haplotypes and schizophrenia. This suggests that AK'T1 does not have an important role in the predisposition to schizophrenia in a Taiwanese population.

\section{Acknowledgements}

We acknowledge the help from the Department of Medical Research in National Taiwan University Hospital and the SNP genotyping work done by the National Genotyping Center.

\section{References}

Bailer U, Leisch F, Meszaros K, Lenzinger E, Willinger U, Strobl R, et al. (2000). Genome scan for susceptibility loci for schizophrenia. Neuropsychobiology 42:175-182.

Chen WJ (1999). Diagnostic interview for genetic studies (DIGS). Mandarin version 2.0 .

Clayton D (1999). A generalization of the transmission/disequilibrium test for uncertain-haplotype transmission. Am J Hum Genet 65: 1170-1177.

Emamian ES, Hall D, Birnbaum MJ, Karayiorgou M, Gogos JA (2004) Convergent evidence for impaired AKT1-GSK3beta signaling in schizophrenia. Nat Genet 36:131-137.

Gabriel SB, Schaffner SF, Nguyen H, Moore JM, Roy J, Blumenstiel B, et al. (2002). The structure of haplotype blocks in the human genome. Science 296:2225-2229.

Horvath S, Xu X, Lake SL, Silverman EK, Weiss ST, Laird NM (2004). Familybased tests for associating haplotypes with general phenotype data: application to asthma genetics. Genet Epidemiol 26:61-69.

Hwu HG (1999). Psychiatric diagnostic assessment. pp. 7-42.

Hwu HG, Chen CH, Hwang TJ, Liu CM, Cheng JJ, Lin SK, et al. (2002). Symptom patterns and subgrouping of schizophrenic patients: significance of negative symptoms assessed on admission. Schizophr Res 56: 105-119.

Hwu HG, Faraone SV, Liu CM, Chen WJ, Liu SK, Shieh MH, et al. (2005). Taiwan schizophrenia linkage study: the field study. Am J Med Genet $B$ Neuropsychiatr Genet 134:30-36.

Ikeda M, Iwata N, Suzuki T, Kitajima T, Yamanouchi Y, Kinoshita Y, et al. (2004). Association of AKT1 with schizophrenia confirmed in a Japanese population. Biol Psychiatry 56:698-700.

Kozlovsky N, Shanon-Weickert C, Tomaskovic-Crook E, Kleinman JE, Belmaker RH, Agam G (2004). Reduced GSK-3beta mRNA levels in postmortem dorsolateral prefrontal cortex of schizophrenic patients. J Neural Transm 111:1583-1592.

O'Connell JR, Weeks DE (1998). PedCheck: a program for identification of genotype incompatibilities in linkage analysis. Am J Hum Genet 63 259-266.

Ohtsuki T, Inada T, Arinami T (2004). Failure to confirm association between AKT1 haplotype and schizophrenia in a Japanese case-control population. Mol Psychiatry 9:981-983.

SAS Institute (2002). SAS/Genetics user's guide. Cary, North Carolina: SAS Institute Inc.

Staal SP (1987). Molecular cloning of the akt oncogene and its human homologues AKT1 and AKT2: amplification of AKT1 in a primary human gastric adenocarcinoma. Proc Natl Acad Sci USA 84: 5034-5037.

Terwilliger JD, Ott J (1994). Handbook of human genetic linkage. 